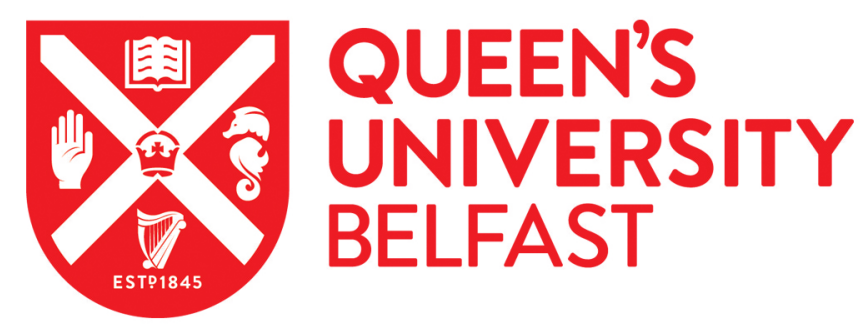

\title{
Sterically stabilized liposomes production using staggered herringbone micromixer: Effect of lipid composition and PEG-lipid content
}

Cheung, C. C. L., \& Al-Jamal, W. T. (2019). Sterically stabilized liposomes production using staggered herringbone micromixer: Effect of lipid composition and PEG-lipid content. International Journal of Pharmaceutics, 566, 687-696. https://doi.org/10.1016/j.ijpharm.2019.06.033

\section{Published in:}

International Journal of Pharmaceutics

\section{Document Version:}

Peer reviewed version

Queen's University Belfast - Research Portal:

Link to publication record in Queen's University Belfast Research Portal

\section{Publisher rights}

Copyright 2019, Elsevier.

This work is made available online in accordance with the publisher's policies. Please refer to any applicable terms of use of the publisher.

\section{General rights}

Copyright for the publications made accessible via the Queen's University Belfast Research Portal is retained by the author(s) and / or other copyright owners and it is a condition of accessing these publications that users recognise and abide by the legal requirements associated with these rights.

Take down policy

The Research Portal is Queen's institutional repository that provides access to Queen's research output. Every effort has been made to ensure that content in the Research Portal does not infringe any person's rights, or applicable UK laws. If you discover content in the Research Portal that you believe breaches copyright or violates any law, please contact openaccess@qub.ac.uk. 


\section{Sterically stabilized liposomes production using staggered herringbone micromixer: Effect of lipid composition and PEG-lipid content}

Calvin C.L. Cheung and Wafa T. Al-Jamal

5 School of Pharmacy, Queen's University Belfast, Belfast, United Kingdom

*Corresponding author:

Dr Wafa' T. Al-Jamal

School of Pharmacy

Queen's University Belfast

10 Belfast, BT9 7BL

United Kingdom

E-mail:w.al-jamal@qub.ac.uk

Telephone: $+44(0) 2890972609$

15 KEYWORDS: Doxorubicin, Liposomes, Microfluidics, PEGylated liposomes, Staggered Herringbone Micromixer 


\section{Abstract}

Preparation of lipid-based drug delivery systems by microfluidics has been increasingly

20 popular, due to the reproducible, continuous and scalable nature of the microfluidic process. Despite exciting development in the field, versatility and superiority of microfluidics over conventional methods still need further evidence, since preparing clinically-relevant sterically stabilised liposomes has been lacking. The present study describes the optimisation of PEGylated liposomal formulations of various rigidity using staggered herringbone micromixer (SHM). The effect of both processing parameters (total flow rate (TFR) and aqueous-to-ethanol flow rate ratio (FRR)) and formulation parameters (lipid components and composition, initial lipid concentration and aqueous media) was investigated and discussed. Liposomal formulations consist of 1,2-dioleoyl-sn-glycero-3-phosphatidylcholine (DOPC), 1,2dipalmitoyl-sn-glycero-3-phosphocholine (DPPC) or 1,2-distearoyl-sn-glycero-3-

30 phosphatidylcholine (DSPC), with cholesterol and PEGylated lipid (DSPE-PEG 2000 ) were successfully prepared with the desired size $(\sim 100 \mathrm{~nm})$ and dispersity $(<0.2)$. Doxorubicin was successfully encapsulated in these liposomes at high (> 80\%) encapsulation efficiency using the pH-gradient remote loading method, illustrating their bilayer integrity and capability as drug delivery systems. We demonstrated that clinically-relevant PEGylated liposomal formulations could be prepared with properties comparable to conventional techniques. Limitations and recommendations on the microfluidic production of PEGylated liposomes were also discussed. 


\section{Introduction}

40 Liposomes are enclosed phospholipid vesicles that have been clinically approved to encapsulate a wide range of therapeutics and diagnostics (Bulbake et al., 2017; Chang and Yeh, 2012; Cheung and Al-Jamal, 2018; Kraft et al., 2014). Microfluidics has been explored to prepare liposomes in a well-controlled, reproducible and high-throughput manner; thereby overcoming hurdles of conventional techniques, namely low batch-to-batch reproducibility and

45 limited throughput (Björnmalm et al., 2014; Lu et al., 2016; Ma et al., 2017; Maeki et al., 2018; Valencia et al., 2012). An exciting recent advancement was the development of staggered herringbone micromixer (SHM) that showed superiority (higher throughput, faster mixing and lesser dilution) over the conventional microfluidic hydrodynamic focusing (MHF) devices (Stroock, 2002; Zhigaltsev et al., 2012).

50 Currently, there are two obstacles impeding the progression of microfluidics as the preferable technique over the conventional techniques: the hurdles for researchers without prior microfluidic knowledge and experience to design, select and operate microfluidic devices (Björnmalm et al., 2014); and concerns about whether microfluidics could meet their specific need, as conventional methods have been able to provide (Carugo et al., 2016; Sackmann et al., 2014; Whitesides, 2013). The former have been alleviated by commercialisation of microfluidic devices (Volpatti and Yetisen, 2014), while the latter could be resolved by demonstrating functionality of microfluidics, compared to conventional methods, in preparing clinically-relevant liposomal formulations (Sackmann et al., 2014; Whitesides, 2013).

To date, most clinically approved (and under clinical trial) liposomal formulations are sterically stabilised, with 100-150 nm diameter (Bulbake et al., 2017; Chang and Yeh, 2012; Kraft et al., 2014; Suk et al., 2016). PEGylated liposomes, compared to non-PEGylated liposomes, have shown to exhibit increased stability, reduced dispersity and prolonged blood circulation time 
(Suk et al., 2015). The clinical significance of PEGylation has been demonstrated by the inclusion of PEG-lipid in the clinically-approved liposomal formulations: Doxil ${ }^{\circledR}$ and Onivyde $^{\circledR}$ (Bulbake et al., 2017). Despite the high demands for sterically stabilised drug delivery systems, most existing microfluidics studies (not limited to SHM) reported the production of non-PEGylated formulations (Forbes et al., 2019; Guimarães Sá Correia et al., 2017; Kastner et al., 2015; Maeki et al., 2015; Zhigaltsev et al., 2012). Few studies reported the preparation of PEGylated liposomes, which were either very small in size $(\sim 50 \mathrm{~nm})$, unstable or of high dispersity (> 0.2) (Dong et al., 2017; Hood et al., 2014; Ran et al., 2016; Zheng and Fyles, 2018; Zhigaltsev et al., 2015; Zizzari et al., 2017). For instance, Zhigaltsev et al. failed to produce stable and monodispersed, high phase-transition liposomes (DPPC or HSPC) using SHM microfluidics, and mixing with unsaturated lipids was needed to enhance the stability of these PEGylated liposomes (Zhigaltsev et al., 2015). Furthermore, majority of these reports investigated only the effect of total flow rate (TFR) and aqueous-to-ethanol flow rate ratio (FRR) on liposome quality (Guimarães Sá Correia et al., 2017; Jahn et al., 2008; Kastner et al., 2014; Zhigaltsev et al., 2012), since they are the only processing parameters for liposomes production. Such approach might have demonstrated the robustness and simplicity of microfluidics, where optimisation of nanoparticles preparation could be achieved by simply controlling TFR and FRR. On the other hand, this might indicate our lack of knowledge on the limitation of this technique to prepare liposomes. Namely, formulations that could not be prepared, with an optimal size and dispersity, solely by optimising the processing parameters.

To date, here have been few studies which reported, individually, the effect of formulation parameters: choice of aqueous and/or organic medium (Joshi et al., 2016; Obeid et al., 2017); lipid concentration (Joshi et al., 2016; Maeki et al., 2017); lipid components (Zhigaltsev et al., 2015) and composition (Hood et al., 2013) on nanoparticles preparation. However, there are contradictory results regarding the effect of some of the parameters, possibly due to the 
difference in microfluidic devices and range of values investigated, leading to difficulty in making conclusive comparison. Encouragingly, a recent study by Forbes et al. investigated using SHM the effect of preparation temperature, lipid concentration, lipid components and composition on the liposome size and dispersity of non-PEGylated liposomes (Forbes et al., 2019). Herein we have demonstrated successfully the preparation of PEGylated liposomal formulations with the desired size $(\sim 100 \mathrm{~nm})$ and dispersity $(<0.2)$ using SHM. We systematically studied the effect of formulation parameters (aqueous media, initial lipid concentration, lipid components and composition), besides the processing parameters (TFR and FRR). To the best of our knowledge, this is the first report of producing stable, monodispersed ( 100 nm), PEGylated liposomes using SHM, including both fluid and rigid liposomal formulations, where the latter are more clinically relevant. 


\section{Materials and Methods}

\subsection{Materials}

1,2-dioleoyl-sn-glycero-3-phosphatidylcholine （DOPC), 1,2-dipalmitoyl-sn-glycero-3phosphocholine (DPPC), 1,2-distearoyl-sn-glycero-3-phosphatidylcholine (DSPC), 1,2distearoyl-sn-glycero-3-phosphoethanolamine-N-[methoxy(polyethylene glycol)-2000]

105 (DSPE-PEG 2000 ), were generous gifts from Lipoid GmbH (Ludwigshafen, Germany). Doxorubicin hydrochloride (DOX) was purchased from Apollo Scientific (Cheshire, UK). Ammonium sulfate $\quad\left(\left(\mathrm{NH}_{4}\right)_{2} \mathrm{SO}_{4}\right), \quad$ cholesterol $\quad(\mathrm{Chol}), \quad$ 4-(2-hydroxyethyl)-1piperazineethanesulfonic acid (HEPES), phosphate buffered saline (PBS) tablets, sodium chloride $(\mathrm{NaCl})$, Triton ${ }^{\mathrm{TM}} \mathrm{X}-100$ were purchased from Sigma-Aldrich (Dorset, UK). Ethanol 110 absolute was purchased from VWR (Leicestershire, UK).

\subsection{Preparation of liposomes using microfluidics}

SHM (Darwin Microfluidics, Paris, France) was used as the microfluidic device to prepare liposomes. Lipids were dissolved in ethanol to prepare lipid mixture of appropriate concentration and molar ratio. Aqueous media used to prepare the liposomes included

115 deionised water (DW), isotonic normal saline (NS; $0.9 \% \mathrm{w} / \mathrm{v} \mathrm{NaCl}$ ), phosphate buffered saline (PBS; $10 \mathrm{mM}$ phosphate, $0.8 \% \mathrm{w} / \mathrm{v} \mathrm{NaCl}, \mathrm{pH} 7.4$ ), HEPES buffered saline (HBS; $20 \mathrm{mM}$ HEPES, $0.8 \%$ w/v NaCl, pH 7.4) and ammonium sulfate (( $\left.\left.\mathrm{NH}_{4}\right)_{2} \mathrm{SO}_{4} ; 240 \mathrm{mM}, \mathrm{pH} 5.4\right)$. All aqueous media were passed through $0.22 \mu \mathrm{m}$ polyether sulfone syringe filter before injecting into the SHM.

120 Ethanol solution of lipid mixture and aqueous medium were injected into the SHM with their volume flow rates controlled by two syringe pumps (AL1000-220, World Precision 
Instruments, Hertfordshire, UK) using SyringePumpPro software. The experiment setup is as illustrated in Scheme 1. For formulations composed of phospholipids of high melting point (DPPC and DSPC), a heating tape (Omega Engineering Ltd., Manchester, UK) was used to maintain the temperature of both ethanol and aqueous solutions at least $10{ }^{\circ} \mathrm{C}$ above the gelto-liquid-crystalline phase transition temperature of the phospholipid $\left(51{ }^{\circ} \mathrm{C}\right.$ for DPPC and 65 ${ }^{\circ} \mathrm{C}$ for DSPC). Liposomes were collected from the outlet of the SHM and ethanol was removed by dialysis overnight at room temperature, against $1 \mathrm{~L}$ of the same aqueous medium used in the preparation, under constant stirring, using Pur-A-Lyzer ${ }^{\mathrm{TM}}$ Dialysis Kit (12 kDa molecular

130 weight cut-off; Sigma-Aldrich, Dorset, UK).

\section{Scheme 1}

\subsection{DOX loading into liposomes using the pH-gradient remote loading method}

DOX was loaded into liposomes using a pH-gradient remote loading method. Liposomes were first prepared in ammonium sulfate as the aqueous medium. Following dialysis, the external

135 buffer of the liposomes was exchanged to HBS using PD-10 Sephadex G-25 gel filtration column (GE Healthcare Life Sciences, Buckinghamshire, UK). The liposomes were incubated with DOX at drug-to-phospholipid (excluding cholesterol) molar ratio of 1:20 for 1 hour, at room temperature, $45^{\circ} \mathrm{C}$ and $60{ }^{\circ} \mathrm{C}$ for DOPC-, DPPC-, DSPC-based liposomes, respectively. After the incubation, liposomes were purified by removing unencapsulated DOX using PD-10

140 gel filtration column, as described above. To quantify the encapsulation efficiency (EE) of DOX, liposomes before and after purification were diluted to the same lipid concentration and then solubilised by Triton X-100 to release encapsulated DOX. A final concentration of $0.1 \mathrm{v} / \mathrm{v}$ \% Triton X-100 was used, corresponds to phospholipid-to-detergent molar ratio of 1:20, sufficient to ensure complete solubilisation of liposomes (Dennis, 1974; López et al., 1998). 
145 DOX fluorescence intensity was measured using FLUOstar Omega Microplate Reader (BMG LABTECH Ltd., UK) with excitation wavelength of $485 \mathrm{~nm}$ and emission wavelength of 590 $\mathrm{nm}$. The concentration of DOX in the wells were within the linear region. EE of DOX was then calculated by comparing the fluorescence intensity of the samples before and after purification:

$$
\text { Encapsulation efficiency }(\%)=\frac{\mathrm{I}(\mathrm{t}) \text { after purification }}{\mathrm{I}(\mathrm{t}) \text { before purification }} \times 100
$$

\subsection{Particle size and size distribution}

Particle size and size distribution of the liposomes were characterised by their Z-average diameter and dispersity by dynamic light scattering (DLS), using Zetasizer Nano ZS90 (Malvern Panalytical, Worcestershire, UK) equipped with a $4.0 \mathrm{~mW}$ He-Ne laser operating at $633 \mathrm{~nm}$ with photodiode detector at a detection angle at $90^{\circ}$. The term "dispersity" is used instead of "polydispersity index", in accordance with recommendations of IUPAC (Stepto, 2009). Samples were diluted 10-fold in DW and loaded in a low-volume polystyrene cuvette. Z-average and dispersity of each sample were obtained as the average of three measurements. The zeta potential $(\zeta)$ was obtained by measuring the particle mobility using phase analysis light scattering. Samples were diluted 10 -fold in DW and loaded in a folded capillary cell. Six measurements were performed for each sample at $25^{\circ} \mathrm{C}$. Zeta potential of each sample was obtained as the average of six measurements.

\subsection{Transmission electron microscopy (TEM)}

The morphology of the microfluidic-prepared liposomes was studied using a JEOL JEM-1400 plus transmission electron microscope, operating at an accelerating voltage of $120 \mathrm{kV}$. Samples 
165 for the TEM analysis were prepared by adding $5 \mu \mathrm{L}$ of liposome suspension on a carbon-coated 400 mesh copper grid (Ted Pella, Inc., Redding, CA, US) and allowed to air-dry. Negative stain was applied by adding $5 \mu \mathrm{L}$ of $1 \%$ (w/v) phosphotungstic acid (pH 7.0 adjusted with $\mathrm{NaOH}$ ) and allowed to air-dry.

\subsection{Statistical analysis}

170 Student's unpaired two-tailed t-test and one-way analysis of variance (ANOVA) followed by Fisher's least significant difference (LSD) test were used to assess statistical significance between group means, with the significance level $\alpha=0.05$ (Rothman, 1990; Saville, 1990). Regression analyses were performed using least-squares fitting. All analyses were performed in GraphPad Prism 7.0 (GraphPad Software Inc., CA, US)

175 


\section{Results}

In the present work, three phospholipids (DOPC, DPPC and DSPC) with different phase transition were used to formulate sterically stabilised liposomes. To enhance the stability of our formulations, all liposomes contained $50 \mathrm{~mol} \%$ cholesterol with respect to phospholipid

180 (Briuglia et al., 2015), and up to 5 mol\% DSPE-PEG 2000 to prolong their blood circulation (Garbuzenko et al., 2005; Kenworthy et al., 1995). All the formulations prepared in this study are listed in Table 1. The initial lipid concentration was $10 \mathrm{mM}$, unless otherwise stated. Isotonic normal saline (NS) and phosphate buffered saline (PBS) were used as the aqueous media, and ethanol was selected as the organic solvent, due to its relatively low toxicity 185 (Church and Witting, 1997).

\section{Table 1}

In the first part of our investigation, we studied the effect of process parameters: total flow rate (TFR) and aqueous-to-ethanol flow rate ratio (FRR) on the size and dispersity of a fluidic PEGylated liposomal formulation (DOPC5). This was followed by the investigation of the

190 effect of aqueous medium, and initial lipid concentration on the production of DOPC5. We then optimised the preparation of unprecedented $100 \mathrm{~nm}$, sterically stabilised, high phase transition DSPC liposomes by manipulating the formulation parameters (i.e. DSPE-PEG 2000 content), besides the processing parameters. Finally, doxorubicin (DOX) was actively loaded into these PEGylated liposomes by the ammonium sulfate gradient method (Haran et al., 1993) to evaluate their capability for drug loading. 


\subsection{Preparation of DOPC5 liposomes using microfluidics}

DOPC is an unsaturated phospholipid consists of two oleyl hydrocarbon chains (18 carbon atoms with one cis double bond at omega-9 position), which has a phase transition temperature of $-17^{\circ} \mathrm{C}$. This implies that DOPC is already at liquid-crystalline phase at room temperature.

200 The effect of aqueous medium on liposome size and dispersity was investigated in the present work. The organic (10 mM DOPC5 in ethanol) and aqueous phases (DW, NS and PBS) were injected into the SHM with TFR of 1 and $2 \mathrm{~mL} / \mathrm{min}$ and FRR of 1. The Z-average diameter and dispersity of the liposomes prepared are shown in Figure 1.

\section{Figure 1}

205 The differences in size and dispersity between liposomes prepared in DW compared to NS and PBS were obvious. A one-way ANOVA was conducted to compare the effect of the three aqueous media on DOPC5 size or dispersity. At TFR $1 \mathrm{~mL} / \mathrm{min}$, there was a significant effect of aqueous medium on $\mathrm{Z}$-average $\left(\mathrm{F}_{2,6}=11.81, \mathrm{p}=0.008\right)$, where the size of liposomes prepared in DW $(69.38 \mathrm{~nm})$ was significantly smaller than those prepared in NS $\left(102.0 \mathrm{~nm}\right.$; $\mathrm{t}_{6}$

$210=4.455, \mathrm{p}=0.0043)$ and PBS $\left(97.98 \mathrm{~nm} ; \mathrm{t}_{6}=3.91, \mathrm{p}=0.0079\right)$. The dispersity of the liposomes was also significantly influenced by the aqueous medium $\left(F_{2,6}=11.77, p=0.008\right)$, where liposomes prepared in DW showed significantly higher dispersity (0.219) compared to those prepared in NS $\left(0.095 ; \mathrm{t}_{6}=4.591, \mathrm{p}=0.0037\right)$ and PBS $\left(0.121 ; \mathrm{t}_{6}=3.653, \mathrm{p}=0.0107\right)$. However, the difference of preparing in NS and PBS was not significant for both size and

215 dispersity. Similarly, at TFR of $2 \mathrm{~mL} / \mathrm{min}$, there were significant effects on both Z-average $\left(\mathrm{F}_{2,6}=9.679, \mathrm{p}=0.013\right)$ and dispersity $\left(\mathrm{F}_{2,6}=74.64, \mathrm{p}<0.001\right)$. It was evident that the absence of ions in the aqueous media might result in smaller and more dispersed (dispersity $>0.2$ ) liposomes, therefore, DW as an aqueous media was excluded from further studies. 
Next, the effect of TFR and FRR on DOPC5 production was studied. The organic $(10 \mathrm{mM}$ DOPC5) and aqueous phases (NS and PBS) were injected into the SHM with TFR of 1 and 2 $\mathrm{mL} / \mathrm{min}$, and FRR of 1, 2, 3 and 5. The Z-average diameter and dispersity of the prepared liposomes were determined using DLS, as shown in Figure 2.

Figure 2

Our results showed that DOPC5 prepared at the highest ethanol content (FRR 1) exhibited the largest size with a hydrodynamic size of $100 \mathrm{~nm}$. Interestingly, as the ethanol content decreased at FRR of 2, 3 and 5, the liposomes size dramatically and significantly decreased to 30-40 nm. The relationship between ethanol content (FRR) and the Z-Average diameter of DOPC5 were evaluated using linear and exponential regression models (Table S1). An exponential relationship was better fitted for DOPC5 prepared in NS or PBS, and regardless of the TFR (1 or $2 \mathrm{~mL} / \mathrm{min}$ ) used (Figure $\mathrm{S} 1$ ). This suggests that FRR is a determinant factor in controlling size of liposomes produced by microfluidics, as previously reported with other conventional liposomes (Kastner et al., 2014; Maeki et al., 2017). The dispersity of all liposomes was similar $(<0.2)$, demonstrating the size of the prepared liposomes were highly homogenous. TFR of 2 $\mathrm{mL} / \mathrm{min}$ and FRR of 1 were selected as the optimised flow conditions for the DOPC5 formulation using SHM device.

Following the evaluation of the effect of processing parameters (TFR and FRR) on DOPC5 production, the effect of the initial lipid concentration was investigated. The organic $(5,10$ and $15 \mathrm{mM}$ of DOPC5) and aqueous phases (NS and PBS) were injected into the SHM with TFR of $2 \mathrm{~mL} / \mathrm{min}$ and FRR of 1 . Here, the effect of initial lipid concentration was studied, instead 240 of the final lipid concentration, to isolate the effect of FRR; since the final lipid concentration is dependent on both initial lipid concentration and FRR (Figure 3). depicts the hydrodynamic size and dispersity of DOPC5 prepared at different initial concentrations. 
Figure 3

A one-way ANOVA was conducted to study the effect of initial lipid concentration on DOPC5 size or dispersity. For the liposomes prepared in NS, as the initial lipid concentration increased from $5 \mathrm{mM}$ to $15 \mathrm{mM}$, the average size increased slightly from $97.1 \mathrm{~nm}$ to $107.8 \mathrm{~nm}$. There were no statistically significant differences in $\mathrm{Z}$-average $\left(\mathrm{F}_{2,6}=1.498, \mathrm{p}>0.05\right)$ between these formulations. Although there were significant differences in dispersity $\left(F_{2,6}=17.43, p=0.003\right)$, the dispersity of all liposomes was lower than 0.2. Meanwhile, there were no significant differences in both Z-Average $\left(F_{2,8}=3.15, p>0.05\right)$ and dispersity $\left(F_{2,8}=4.266, p>0.05\right)$ for liposomes prepared in PBS. Overall, it was concluded that the initial lipid concentration only has a minor effect on the size and dispersity of DOPC5 prepared using SHM.

\subsection{Microfluidics preparation of high phase transition, sterically stabilised liposomes}

In the second part of our work we moved towards optimising the production of sterically stabilised DSPC liposomes, that are more clinically relevant, such Doxil ${ }^{\circledR}$ and Onivyde ${ }^{\circledR}$ (Bulbake et al., 2017). In contrast to DOPC $\left(\mathrm{T}_{\mathrm{m}}=-17^{\circ} \mathrm{C}\right)$, which exists in a liquid-crystalline phase at room temperature, DSPC $\left(\mathrm{T}_{\mathrm{m}}=55^{\circ} \mathrm{C}\right)$ exhibits in the gel phase which would greatly limit their ability to self-assemble into vesicles properly. Therefore, DSPC-based formulation was prepared at $65^{\circ} \mathrm{C}$, by heating both aqueous and organic phases prior to being injected into the microfluidic device. The organic (10 mM DSPC5) and aqueous phases (NS and PBS) were injected into the SHM at $65{ }^{\circ} \mathrm{C}$ with TFR of $2 \mathrm{~mL} / \mathrm{min}$ and FRR of $2,2.5,3$ and 4 . The Zaverage diameter and dispersity of the prepared liposomes were shown in Figure 4.

Figure 4 
DSPC5 prepared in NS with TFR of $2 \mathrm{~mL} / \mathrm{min}$ followed the expected response towards the change in FRR, where their size decreased quickly with increasing FRR. Starting at an intermediate FRR of 3, DSPC5 had an average size of $93.78 \mathrm{~nm}$ and dispersity of 0.238 , which was fairly dispersed. These liposomes are significantly larger $\left(\mathrm{t}_{7}=5.545, \mathrm{p}<0.001\right)$ and more disperse $\left(\mathrm{t}_{7}=5.532, \mathrm{p}<0.001\right)$ compared to DOPC5 prepared in the same flow conditions (size of $29.56 \mathrm{~nm}$ and dispersity of 0.098 ; Figure 2). As the FRR was reduced to 2.5 and 2, the size of the liposomes increased to $133.9 \mathrm{~nm}$ and $194.2 \mathrm{~nm}$ and the dispersity rose to 0.371 and 0.430 , respectively, with the presence of some precipitates. On the other hand, by increasing the FRR from 3 to 4, the size of the liposomes decreased as expected, to $62.84 \mathrm{~nm}$; however, the sample remained dispersed, with a dispersity of 0.283 . By optimising the FRR, we were able to obtain DSPC5 with the desired size of roughly $100 \mathrm{~nm}$; however, the dispersity, which was relatively unresponsive towards both TFR and FRR (as mentioned above), could not be improved and remained high (>0.2). Similar results were observed with DSPC5 prepared in PBS.

Here we inferred that DSPC5 became increasingly unstable with $>25 \mathrm{v} / \mathrm{v} \%$ ethanol (FRR < 3); in comparison to DOPC5, liposomes remained stable with $50 \mathrm{v} / \mathrm{v} \%$ ethanol (FRR of 1, Figure 1). In addition, it is worth mentioning that peaks in the micron range were reported by DLS in both intensity- (Figure S2, left) and volume-weighted size distribution (Figure S2, right) for all the above flow conditions (TFR of $2 \mathrm{~mL} / \mathrm{min}$ and FRR of $2-4$ ), signifying the presence and contribution of these peaks, respectively. While the distribution (width) of the main peak remained relatively similar, the proportion of the micron-sized peak decreased sharply with decreasing ethanol concentration (i.e. increasing FRR). Nevertheless, the micron-sized peak was present even at $20 \mathrm{v} / \mathrm{v} \%$ ethanol (FRR of 4 ).

Although the presence of micron-sized peaks in a DLS report does not necessary imply the presence of micron-sized particles, it suggests the presence of certain species with slow Brownian motion or diffusivity, which is unlikely to be of the same liposome population. This 
result suggested that the DSPC5 formulation per se would not self-assemble homogeneously, without size homogenisation procedures such as membrane extrusion (Pereira et al., 2016). Together with the aforementioned effect of processing parameters (TFR and FRR) on size and dispersity, it could be extrapolated that further optimisation of processing parameters would not produce DSPC5 the desired size $(\sim 100 \mathrm{~nm})$ and dispersity $(<0.2)$.

Since the production of DSPC5 with a monomodal size distribution was not achievable, the formulation was modified by reducing the amount of DSPE-PEG ${ }_{2000}$. Namely, non-PEGylated formulation of DSPC0 and PEGylated formulation of DSPC2.5, DSPC4 and DSPC5 were prepared. The organic $(10 \mathrm{mM})$ and aqueous phases (NS and PBS) were injected into the SHM at $65{ }^{\circ} \mathrm{C}$ with our previously optimised TFR of $2 \mathrm{~mL} / \mathrm{min}$ and FRR of 3 . The Z-average diameter and dispersity of the liposomes prepared in NS and PBS are shown in Figure 5.

Interestingly, our results showed that reducing the DSPE-PEG 2000 content produced larger and more homogenous liposomes. A one-way ANOVA was conducted to study the effect of DSPE$\mathrm{PEG}_{2000}$ content on DSPC/Chol/DSPE-PEG $2000(100-\mathrm{x} / 50 / \mathrm{x}, \mathrm{x}=0-5)$ liposome size or dispersity. There were statistically significant differences in both Z-Average $\left(F_{3,16}=62.15, p\right.$ $<0.001)$ and dispersity $\left(\mathrm{F}_{3,16}=16.88, \mathrm{p}<0.001\right)$ between these liposomes prepared in NS. As we decreased DSPE-PEG 2000 from 5 to 4 mol\%, we observed immediate improvement with the dispersity. The size of DSPC4 liposomes changed minimally from 93.78 to $93.19 \mathrm{~nm}\left(\mathrm{t}_{16}=\right.$ $0.0854, \mathrm{p}>0.05)$, but the dispersity reduced from 0.238 to $0.143\left(\mathrm{t}_{16}=4.381, \mathrm{p}<0.001\right)$, with the presence of the micron-sized peak (Figure S3). Further reduction in the DSPE-PEG 2000 310 content to $2.5 \mathrm{~mol} \%$, liposomes size of DSPC2.5 increased to an average of $122.0 \mathrm{~nm}\left(\mathrm{t}_{16}=\right.$ $3.246, \mathrm{p}=0.005)$. Unexpectedly, the dispersity decreased significantly down to $0.055\left(\mathrm{t}_{16}=\right.$ $6.632, \mathrm{p}<0.001$ ), and the micron-sized peak was no longer reported, as shown in Figure S3. 
Thus, DSPC2.5 was deemed the optimal DSPC-based PEGylated liposomal formulation. Similar results and significance difference (Z-Average: $F_{2,7}=6.162, p=0.0286$ and dispersity:

$\left.315 \mathrm{~F}_{2,7}=9.303, \mathrm{p}=0.0107\right)$ were observed using PBS. Upon removing $\mathrm{DSPE} \mathrm{PEG}_{2000}$ from the formulation (DSPC0), the size further increased to $189.4 \mathrm{~nm}\left(\mathrm{t}_{16}=12.04, \mathrm{p}<0.001\right)$ and the dispersity increased to $0.204\left(\mathrm{t}_{16}=1.357, \mathrm{p}>0.05\right)$, with the presence of the micron-sized peak. However, in contrast to DSPC5, it was possible to prepare smaller DSPC0 of $131.6 \mathrm{~nm}$ (dispersity of 0.102 ) and $86.7 \mathrm{~nm}$ (dispersity of 0.128 ) with FRR of 4 and 5, respectively, as shown in Figures S4 \& S5. Similar results were obtained with liposomes prepared in PBS.

Next, we replaced DSPC with DPPC, a saturated phospholipid consists of two hydrocarbon chains with 16 carbon atoms (instead of 18 carbon atoms as in DSPC), which has a phase transition temperature of $41{ }^{\circ} \mathrm{C}$. The organic (10 mM DPPC5) and aqueous phases (NS and PBS) were injected into the $\mathrm{SHM}$ at $51^{\circ} \mathrm{C}$ with TFR of $2 \mathrm{~mL} / \mathrm{min}$ and FRR of 3 (Figure 6).

Figure 6

The average size and dispersity of the prepared DPPC5 in NS were $107.4 \mathrm{~nm}$ and 0.099 . respectively. A one-way ANOVA was conducted to study the effect of phospholipid rigidity on liposome size or dispersity. Unexpectedly, there were statistically significant differences in both Z-Average $\left(\mathrm{F}_{2,9}=25.97, \mathrm{p}<0.001\right)$ and dispersity $\left(\mathrm{F}_{2,9}=28.00, \mathrm{p}<0.001\right)$ between DOPC5, DPPC5 and DSPC5 prepared in NS. Compared to the rigid DSPC5 formulation, the difference in size was not significant ( $\left.\mathrm{t}_{9}=1.321, \mathrm{p}>0.05\right)$, but the dispersity of the liposomes was significantly lower $\left(t_{9}=6.103, p<0.001\right)$. Furthermore, no micron-sized peak was reported. Similar results and significance difference (Z-Average: $F_{2,7}=80.03, p<0.001$ and dispersity: 
$\left.\mathrm{F}_{2,7}=14.09, \mathrm{p}<0.01\right)$ were observed using PBS. Since the size and dispersity of DPPC5 prepared with this condition were acceptable, no further optimisation was not performed. This clearly indicates that slight modifications in the composition of the liposomal formulation, such the hydrocarbon length or DSPE-PEG 2000 content could have a major impact on stealth liposomal production using microfluidics.

\subsection{Long-term stability of sterically stabilised liposomes prepared in different aqueous media}

Our results demonstrated the successful preparation of different sterically stabilised liposomal formulations with suitable size and dispersity using SHM microfluidics. Next, to assess their capacity as drug delivery systems, all formulations were prepared in different aqueous media, namely, NS, PBS (pH 7.4), HBS (pH 7.4) and $\left(\mathrm{NH}_{4}\right)_{2} \mathrm{SO}_{4}(\mathrm{pH} 5.4)$, with the respectively optimised flow conditions, as shown in Table 2. HBS and $\left(\mathrm{NH}_{4}\right)_{2} \mathrm{SO}_{4}$ were used for their relevance with drug loading and biological applications. All liposomes were successfully prepared with a hydrodynamic diameter around $100 \mathrm{~nm}$ and dispersity lower than 0.2 (with the exception of DSPC5 which homogenous liposomes could not be obtained). All dialysed liposomes, including DSPC5, were subsequently stored at $4{ }^{\circ} \mathrm{C}$ were stable in size up to 28 days with minimum changes in their size and dispersity (Figure S6).

Table 2

\subsection{Structure elucidation and DOX loading into microfluidic prepared liposomes}

355 The morphology of the three sterically stabilised formulations prepared by their respective optimised flow conditions was characterised using TEM. As shown in Figure 7, all liposomes exhibited vesicular structures and were homogenous in size, with a mean diameter of about 
$100 \mathrm{~nm}$, which is in a good agreement with the Z-average diameter provided by DLS (Table 2).

Once the flow conditions were optimised, the initial lipid concentrations were increased to counteract the dilution by the aqueous media (FRR) to achieve a final concentration of $7.5 \mathrm{mM}$. The vesicular structure and integrity of the lipid bilayer of the microfluidic prepared liposomes, including DSPC5, was confirmed by high encapsulation efficiency of DOX, using the $\mathrm{pH}$ 365 gradient method (Table 3).

Table 3 


\section{Discussion}

370 Steric stabilisation of liposomes by PEGylation prevents liposomes aggregation, enhances stability, and increases their blood circulation half-life (Danhier et al., 2010; Gabizon et al., 1997; Immordino et al., 2006). PEGylation is therefore an essential factor in designing drug delivery systems, as demonstrated in clinically approved formulations Doxil ${ }^{\circledR}$ and Onivyde ${ }^{\circledR}$ (Bulbake et al., 2017) or to achieve active targeting. However, to date, majority of the studies with microfluidics developed non-PEGylated conventional liposomes (Guimarães Sá Correia et al., 2017; Joshi et al., 2016; Kastner et al., 2015; Maeki et al., 2017; Zhigaltsev et al., 2015). In this work, we successfully prepared PEGylated formulations with different rigidity (DOPC5, DPPC5 and DSPC2.5) using SHM, through the optimisation of both processing and formulation parameters. Self-assembly of liposomes in microfluidics is a bottom-up nanoprecipitation technique, thus the liposome quality will depend largely on the physical properties of the liposomal formulation, and the surrounding solvent environment (Capretto et al., 2013). In contrast to conventional bulk production of liposomes, microfluidic techniques do not require post-processing procedures (Yu et al., 2009). Consequently, as the liposomal formulation changes, the optimal flow conditions might have to be changed, as we demonstrated in Table 2, to obtain liposomes of same size and dispersity.

\section{1 [TFR/FRR effect]}

As our results have shown, FRR was the governing parameter controlling the liposome size. Increasing the FRR resulted in sharp decrease in liposome size, until the size approached the lower limit where the difference became gradual (Figure 2). TFR has similar effect on liposome size qualitatively, but the decrease in liposome size and increase in dispersity were instead minimal. The responses in liposome size toward varying TFR and FRR has been reported 
extensively, in both MHF and SHM devices. Encouragingly, the effect of TFR and FRR on liposome size was universal regardless of the type of liposomal formulations (Carugo et al., 2016; Forbes et al., 2019; Jahn et al., 2007; Kastner et al., 2014; Ma et al., 2017). Their effect could be explained by the nanoprecipitation mechanism (Capretto et al., 2013; Maeki et al., 2017). Briefly, as lipid molecules diffuse from solvent (ethanol) to non-solvent (aqueous medium), they become less soluble and form self-assembled intermediate known as bilayered phospholipid fragments (BPF) will be formed (Lasic and Martin, 1990). At lower FRR, the duration for lipids to diffuse into the aqueous non-solvent is extended, allowing BPF to grow 400 larger in size before their eventual closure into larger liposomes; while at higher FRR, BPF are closed into smaller liposomes as they are rapidly exposed to the non-solvent. The critical ethanol concentration for formation of BPF, for unsaturated phospholipid, was estimated to be $80 \%$ ethanol, and their closure into liposomes at $60 \%$ ethanol (Maeki et al., 2017). This result agrees with the optimisation of DOPC5, where liposomes could be formed at 50\% ethanol 405 (FRR of 1).

Interestingly, it is worth mentioning that DOPC5 prepared using SHM were consistently smaller in size with lower dispersity $(\sim 0.1)$ than the ones prepared using "off-the-shelf" microfluidic devices: T-junction and X-junction (Bottaro and Nastruzzi, 2016; Jeffs et al., 2005; Kulkarni et al., 2017). Furthermore, SHM liposomes were more responsive to the change in 410 FRR and less susceptible to the change in TFR compared to the junction devices, and smaller and more uniform liposomes were produced (Figure S7). On the other hand, upon increasing the FRR in the junction devices, the liposomes size decreased at a lesser extent and the dispersity increased greatly (Figure S7). This highlight the high relevance of SHM devices to prepare liposomes for drug delivery purposes. 


\section{2 [Aqueous buffer effect]}

The choice of aqueous medium was reported to affect both the size and dispersity of the liposomes, where low ionic strength medium led to smaller and more dispersed liposomes (Joshi et al., 2016; Obeid et al., 2017). This agrees with our results which liposomes prepared in DW were significantly smaller and more dispersity than those containing ions (NS, PBS,

$420 \mathrm{HBS}$ and $\left.\left(\mathrm{NH}_{4}\right)_{2} \mathrm{SO}_{4}\right)$. It was reported that at low ionic strength, the hydration layer (electrical double layer) around a particle is greater (Crommelin, 1984; Sabín et al., 2006), which might result in premature closure of BPF into smaller and more dispersed liposomes. Nonetheless, at high ionic strength (e.g. isotonic) uniform liposomes could be formed; the difference in the buffer composition had minimum effect on liposomes size which enable drug loading using 425 different loading methods (passive and active loading).

\section{3 [Lipid concentration effect]}

Regulating the concentration of liposomes can improve the operation of the downstream assay, avoiding extra procedures such as centrifugation to concentrate the liposomal samples. The introduction of the aqueous medium as a non-solvent is essential to the formation of liposomes

430 by nanoprecipitation, inevitably leads to dilution of the initial lipid solution. In SHM, chaotic advection provided by herringbone grooves allows preparation of homogenous liposomes at high flow rates (in $\mathrm{mL} / \mathrm{min}$ ) and unprecedently low FRR (typically 1 to 5 , with final lipid concentration in $\mathrm{mM}$ range) (Stroock, 2002), in comparison to MHF devices with typical value of TFR in $\mu \mathrm{L} / \mathrm{min}$ range and FRR > 10 (Hood et al., 2014; Jahn et al., 2007; Zizzari et al., 2017;

435 Zook and Vreeland, 2010). Here we have demonstrated by increasing initial lipid concentration, with respect to the optimised FRR for each formulation (up to $30 \mathrm{mM}$ ), we could obtain a final lipid concentration of $7.5 \mathrm{mM}$, comparable to the liposome concentration obtained with thin 
film hydration method (Pereira et al., 2016). Interestingly, increasing initial lipid concentrations resulted in a slight increase in the liposomes size, without affecting the

440 dispersity (Figure 3, Table 3). Maeki et al. reported increasing liposomes size without affecting dispersity, independent of FRR, from initial lipid concentration of $5 \mathrm{mg} / \mathrm{mL}$ to $20 \mathrm{mg} / \mathrm{mL}$ (6.6 $\mathrm{mM}$ to $26.3 \mathrm{mM}$ ), which was in agreement with our results (Figure 3) (Maeki et al., 2017). However, there have been contradictory reports in the literature on the effect of initial lipid concentration. Preparing non-PEGylated formulation using SHM, it was reported that

445 decreasing initial lipid concentration below $3 \mathrm{mg} / \mathrm{mL}(<5 \mathrm{mM})$ led to increasing liposome size and dispersity (Joshi et al., 2016). This effect was again reported by Forbes et al., across initial lipid concentration of $0.3 \mathrm{mg} / \mathrm{mL}$ to $10 \mathrm{mg} / \mathrm{mL}(\sim 0.5 \mathrm{mM}$ to $17 \mathrm{mM})$ (Forbes et al., 2019). It is possible that at the lower range of initial lipid concentration, the formation of liposomes become less frequent and controlled, increasing the likelihood for formation of heterogeneous liposomes. In our experiment, with an initial lipid concentration of $5 \mathrm{mM}$ and FRR of 3, count rates of liposome samples measured by DLS approached the lower recommended limit of detection, suggesting further reduction in initial lipid concentration might lead to inadequate signals for accurate interpretation of DLS measurements.

\section{4 [Component/Rigidity effect]}

455 Besides optimising the production of PEGylated liposomes, our study showed how the rigidity of the lipid bilayer affects the size and dispersity of the liposomes. It was obvious that the fluid formulation (DOPC5) was significantly smaller in size compared to the rigid formulations (DPPC5 and DSPC5), with the same flow conditions (Figure 6), which agrees with Joshi et al. findings using non-PEGylated formulations and SHM (Joshi et al., 2016). Interestingly, despite 460 the two carbons difference between DPPC and DSPC, monodispersed DPPC5 liposomes were produced, while small liposomes with a micron-sized peak was present in DSPC5, and HSPC5 
(Figure S8). This contradicts Zhigaltsev et al. where purely rigid liposomes were not successfully produced, and unstable and large liposomes were obtained (Zhigaltsev et al., 2015). Lipid bilayers with lower rigidity (or bending elasticity modulus), prepared using MHF, have 465 been shown to bend and close into liposomes at a higher rate, yielding smaller liposomes (Zook and Vreeland, 2010). More important, Takechi-Haraya et al. demonstrated using atomic force microscopy (AFM) that the rigidity of cholesterol-containing liposomes with saturated lipids were still higher than those with unsaturated lipids, despite all of them being in liquid-ordered phase (Takechi-Haraya et al., 2016). These results clearly justify the significant difference in

470 size between the fluid (DOPC5) and rigid formulations (DPPC5, DSPC5), despite all formulations being prepared in the liquid-ordered phase.

\section{5 [PEG-lipid content effect]}

The heterogeneity and the micron-sized peak in DSPC5 formulation could not be eliminated by optimising the processing parameters alone (Figure 4); however, this was resolved through

475 optimisation of formulation parameters, namely by lowering the DSPE-PEG 2000 content down to $2.5 \mathrm{~mol} \%$ (Figure 5). Here we observed that increasing the DSPE-PEG 2000 content decreased the liposome size and increased its dispersity. Similar effect of PEG-lipid decreasing the size of lipid nanoparticle and polymeric nanoparticle systems using SHM, have been reported previously (Chen et al., 2014; Morikawa et al., 2018; Yanez Arteta et al., 2018). The properties

480 and origin of the micron-sized peak were not well understood, but based on our results, we observed its dependence on the ethanol content (FRR) (Figure $4 \&$ S2) and concentration of DSPE-PEG $_{2000}$ (Figure $5 \& \mathrm{~S} 3$ ). Ethanol is known to enhance permeability, promote interdigitation of membranes and causes rupture and coalesce of small liposomes $(<200 \mathrm{~nm})$. This effect is more effective on liposomes with longer and saturated carbon chain length 
485 (Adachi et al., 1995; Boni et al., 1993; Komatsu and Okada, 1995; Patra et al., 2006; Simon and McIntosh, 1984; Vanegas et al., 2012).

PEG-lipid with their bulky polymer chain provides higher steric hindrance, which favours high surface curvature, or micellar structure, and it is known to stabilise or induce the formation of bilayer discs (by stabilising the exposed hydrophobic edge) and micelles at high PEG concentration (Johnsson and Edwards, 2003; Rovira-Bru et al., 2002; Zetterberg et al., 2016). For instance, Edwards et al. observed increasing numbers of circular bilayer discs with increasing DSPE-PEG 2000 proportion for DSPC/Chol/DSPE-PEG ${ }_{2000}$, prepared by the lipid film hydration method (Edwards et al., 1997). Although the DSPE-PEG 2000 content in DSPC5 was lower than the estimated critical value of DSPE-PEG 2000 content where open bilayer discs started to form, this could be due to the difference between lipid film hydration and the microfluidic nanoprecipitation method. Nonetheless, the improvement in dispersity by reducing DSPE-PEG 2000 content, from DSPC5 to DSPC2.5, was indisputable (Figure 5). Therefore, it was speculated that the combined effect of ethanol and DSPE-PEG 2000 destabilise the BPF and leading to their closure into irregular liposomes; however, further studies are still warranted.

In the present work, we systematically investigated all factors that could affect the production of sterically stabilised liposomes. Here we proposed a general flowchart, as a qualitative recommendation, for designing and optimising sterically stabilised liposomal formulations using SHM microfluidics (Scheme 2). The flowchart is arranged based on the influence of each parameter on the prepared liposomes and summarised the effects of changing the processing and formulation parameters on the size and the dispersity of the prepared. We have shown that as we optimise a formulation with microfluidics, one would be expected to design each parameter following the downward direction, and optimise each parameter starting from the processing parameters. In case optimising of the processing parameters could not resolve an 
encountered issue, one would have to modify the formulation following the upward direction; and in the worst-case scenario, to change the lipid component.

Scheme 2

\section{Conclusion}

Our study has shown that by manipulating both processing and formulation parameters, all

515 three PEGylated formulations were successfully prepared with the desired size $(\sim 100 \mathrm{~nm})$, dispersity $(<0.2)$ and final lipid concentration $(7.5 \mathrm{mM})$, comparable to those prepared by the conventional thin film hydration with extrusion method (Pereira et al., 2016). The vesicular structures of the optimised liposomes were confirmed by TEM. Remote loading of DOX into the liposomes by $\mathrm{pH}$-gradient further verified their membrane integrity and ability as a drug

520 delivery system. Regardless of the suboptimal characteristics of DSPC5 in terms of dispersity, it was also able to maintain a pH-gradient and have satisfactory DOX encapsulation efficiency. Furthermore, while the unsaturated DOPC5 and saturated DPPC5 formulation could be prepared in a relatively simple manner by varying the processing parameters (TFR and FRR), DSPC5 was dispersed (dispersity > 0.2) regardless of the processing parameters. By optimising 525 the formulation parameters, namely content of DSPE-PEG ${ }_{2000}$, the modified DSPC2.5 formulation was prepared with small diameter and low dispersity. 


\section{Reference}

530 Adachi, T., Takahashi, H., Ohki, K., Hatta, I., 1995. Interdigitated structure of phospholipidalcohol systems studied by x-ray diffraction. Biophys. J. 68, 1850-1855. https://doi.org/10.1016/S0006-3495(95)80361-9

Björnmalm, M., Yan, Y., Caruso, F., 2014. Engineering and evaluating drug delivery particles in microfluidic devices. J. Control. Release 190, 139-149. https://doi.org/10.1016/j.jconrel.2014.04.030

Boni, L.T., Minchey, S.R., Perkins, W.R., Ahl, P.L., Slater, J.L., Tate, M.W., Gruner, S.M., Janoff, A.S., 1993. Curvature dependent induction of the interdigitated gel phase in DPPC vesicles. BBA - Biomembr. 1146, 247-257. https://doi.org/10.1016/00052736(93)90363-5

540 Bottaro, E., Nastruzzi, C., 2016. "Off-the-shelf” microfluidic devices for the production of liposomes for drug delivery. Mater. Sci. Eng. C 64, 29-33. https://doi.org/10.1016/j.msec.2016.03.056

Briuglia, M.L., Rotella, C., McFarlane, A., Lamprou, D.A., 2015. Influence of cholesterol on liposome stability and on in vitro drug release. Drug Deliv. Transl. Res. 5, 231-242. https://doi.org/10.1007/s13346-015-0220-8

Bulbake, U., Doppalapudi, S., Kommineni, N., Khan, W., 2017. Liposomal formulations in clinical use: An updated review. Pharmaceutics 9, 12. https://doi.org/10.3390/pharmaceutics9020012

Capretto, L., Carugo, D., Mazzitelli, S., Nastruzzi, C., Zhang, X., 2013. Microfluidic and lab- 
550 on-a-chip preparation routes for organic nanoparticles and vesicular systems for nanomedicine applications. Adv. Drug Deliv. Rev. 65, 1496-1532. https://doi.org/10.1016/j.addr.2013.08.002

Carugo, D., Bottaro, E., Owen, J., Stride, E., Nastruzzi, C., 2016. Liposome production by microfluidics: potential and limiting factors. Sci. Rep. 6, 25876. https://doi.org/10.1038/srep25876

Chang, H., Yeh, M.-K., 2012. Clinical development of liposome-based drug: formulation , characterization, and therapeutic efficacy. Int. J. Nanomedicine 7, 49-60. https://doi.org/10.2147/IJN.S26766

Chen, S., Tam, Y.K.Y.Y.C., Lin, P.J.C., Leung, A.K.K., Tam, Y.K.Y.Y.C., Cullis, P.R., 2014.

560 Development of lipid nanoparticle formulations of siRNA for hepatocyte gene silencing following subcutaneous administration. J. Control. Release 196, 106-112. https://doi.org/10.1016/j.jconrel.2014.09.025

Cheung, C., Al-Jamal, W.T., 2018. Liposomes-Based Nanoparticles for Cancer Therapy and Bioimaging. pp. 51-87. https://doi.org/10.1007/978-3-319-89878-0_2

565 Church, A.S., Witting, M.D., 1997. Laboratory testing in ethanol, methanol, ethylene glycol, and isopropanol toxicities. J. Emerg. Med. 15, 687-692. https://doi.org/10.1016/S07364679(97)00150-9

Crommelin, D.J.A., 1984. Influence of lipid composition and ionic strength on the physical stability of liposomes. J. Pharm. Sci. 73, 1559-1563. https://doi.org/10.1002/jps.2600731118

Danhier, F., Feron, O., Préat, V., 2010. To exploit the tumor microenvironment: Passive and active tumor targeting of nanocarriers for anti-cancer drug delivery. J. Control. Release 
148, 135-146. https://doi.org/10.1016/j.jconrel.2010.08.027

Dennis, E.A., 1974. Formation and characterization of mixed micelles of the nonionic surfactant Triton X-100 with egg, dipalmitoyl, and dimyristoyl phosphatidylcholines. Arch. Biochem. Biophys. 165, 764-773. https://doi.org/10.1016/0003-9861(74)90305-1

Dong, Y.-D., Tchung, E., Nowell, C., Kaga, S., Leong, N., Mehta, D., Kaminskas, L.M., Boyd, B.J., 2017. Microfluidic preparation of drug-loaded PEGylated liposomes, and the impact of liposome size on tumour retention and penetration. J. Liposome Res. 0, 1-17. https://doi.org/10.1080/08982104.2017.1391285

Edwards, K., Johnsson, M., Karlsson, G., Silvander, M., 1997. Effect of polyethyleneglycolphospholipids on aggregate structure in preparations of small unilamellar liposomes. Biophys. J. 73, 258-266. https://doi.org/10.1016/S0006-3495(97)78066-4

Forbes, N., Hussain, M.T., Briuglia, M.L., Edwards, D.P., Horst, J.H. ter, Szita, N., Perrie, Y., 2019. Rapid and scale-independent microfluidic manufacture of liposomes entrapping protein incorporating in-line purification and at-line size monitoring. Int. J. Pharm. 556, 68-81. https://doi.org/10.1016/j.ijpharm.2018.11.060

Gabizon, A., Goren, D., Horowitz, A.T., Tzemach, D., Lossos, A., Siegal, T., 1997. Longcirculating liposomes for drug delivery in cancer therapy: A review of biodistribution studies in tumor-bearing animals. Adv. Drug Deliv. Rev. 24, 337-344. https://doi.org/10.1016/S0169-409X(96)00476-0

Garbuzenko, O., Barenholz, Y., Priev, A., 2005. Effect of grafted PEG on liposome size and on compressibility and packing of lipid bilayer. Chem. Phys. Lipids 135, 117-129. https://doi.org/10.1016/j.chemphyslip.2005.02.003

595 Guimarães Sá Correia, M., Briuglia, M.L., Niosi, F., Lamprou, D.A., 2017. Microfluidic 
manufacturing of phospholipid nanoparticles: Stability, encapsulation efficacy, and drug release. Int. J. Pharm. 516, 91-99. https://doi.org/10.1016/j.ijpharm.2016.11.025

Haran, G., Cohen, R., Bar, L.K., Barenholz, Y., 1993. Transmembrane ammonium sulfate gradients in liposomes produce efficient and stable entrapment of amphipathic weak bases.

600 Biochim. Biophys. Acta - Biomembr. 1151, 201-215. https://doi.org/10.1016/00052736(93)90105-9

Hood, R.R., DeVoe, D.L., Atencia, J., Vreeland, W.N., Omiatek, D.M., 2014. A facile route to the synthesis of monodisperse nanoscale liposomes using 3D microfluidic hydrodynamic focusing in a concentric capillary array. Lab Chip 14, 2403. https://doi.org/10.1039/c4lc00334a

Hood, R.R., Shao, C., Omiatek, D.M., Vreeland, W.N., Devoe, D.L., 2013. Microfluidic synthesis of PEG- and folate-conjugated liposomes for one-step formation of targeted stealth nanocarriers. Pharm. Res. 30, 1597-1607. https://doi.org/10.1007/s11095-0130998-3

610 Immordino, M.L., Dosio, F., Cattel, L., 2006. Stealth liposomes: Review of the basic science, rationale, and clinical applications, existing and potential. Int. J. Nanomedicine 1, 297315. https://doi.org/10.1023/A:1020134521778

Jahn, A., Reiner, J.E., Vreeland, W.N., DeVoe, D.L., Locascio, L.E., Gaitan, M., 2008. Preparation of nanoparticles by continuous-flow microfluidics. J. Nanoparticle Res. 10, 925-934. https://doi.org/10.1007/s11051-007-9340-5

Jahn, A., Vreeland, W.N., Devoe, D.L., Locascio, L.E., Gaitan, M., 2007. Microfluidic directed formation of liposomes of controlled size. Langmuir 23, 6289-6293. https://doi.org/10.1021/la070051a 
Jeffs, L.B., Palmer, L.R., Ambegia, E.G., Giesbrecht, C., Ewanick, S., MacLachlan, I., 2005. A scalable, extrusion-free method for efficient liposomal encapsulation of plasmid DNA. Pharm. Res. 22, 362-372. https://doi.org/10.1007/s11095-004-1873-z

Johnsson, M., Edwards, K., 2003. Liposomes, Disks, and Spherical Micelles: Aggregate Structure in Mixtures of Gel Phase Phosphatidylcholines and Poly(Ethylene Glycol)Phospholipids. Biophys. J. 85, 3839-3847. https://doi.org/10.1016/S00063495(03)74798-5

Joshi, S., Hussain, M.T., Roces, C.B., Anderluzzi, G., Kastner, E., Salmaso, S., Kirby, D.J., Perrie, Y., 2016. Microfluidics based manufacture of liposomes simultaneously entrapping hydrophilic and lipophilic drugs. Int. J. Pharm. 514, 160-168. https://doi.org/10.1016/j.ijpharm.2016.09.027

630 Kastner, E., Kaur, R., Lowry, D., Moghaddam, B., Wilkinson, A., Perrie, Y., 2014. Highthroughput manufacturing of size-tuned liposomes by a new microfluidics method using enhanced statistical tools for characterization. Int. J. Pharm. https://doi.org/10.1016/j.ijpharm.2014.10.030

Kastner, E., Verma, V., Lowry, D., Perrie, Y., 2015. Microfluidic-controlled manufacture of liposomes for the solubilisation of a poorly water soluble drug. Int. J. Pharm. 485, 122130. https://doi.org/10.1016/j.ijpharm.2015.02.063

Kenworthy, A.K., Simon, S.A., McIntosh, T.J., 1995. Structure and phase behavior of lipid suspensions containing phospholipids with covalently attached poly(ethylene glycol). Biophys. J. 68, 1903-1920. https://doi.org/10.1016/S0006-3495(95)80368-1

640 Komatsu, H., Okada, S., 1995. Ethanol-induced aggregation and fusion of small phosphatidylcholine liposome: participation of interdigitated membrane formation in their processes. BBA - Biomembr. 1235, 270-280. https://doi.org/10.1016/0005- 
2736(95)80014-7

Kraft, J.C., Freeling, J.P., Wang, Z., Ho, R.J.Y., 2014. Emerging research and clinical 645 development trends of liposome and lipid nanoparticle drug delivery systems. J. Pharm. Sci. 103, 29-52. https://doi.org/10.1002/jps.23773

Kulkarni, J.A., Tam, Y.Y.C.K., Chen, S., Tam, Y.Y.C.K., Zaifman, J., Cullis, P.R., Biswas, S., 2017. Rapid synthesis of lipid nanoparticles containing hydrophobic inorganic nanoparticles. Nanoscale 9, 13600-13609. https://doi.org/10.1039/C7NR03272B

650 Lasic, D.D., Martin, F.J., 1990. On the mechanism of vesicle formation. J. Memb. Sci. 50, 215222. https://doi.org/10.1016/S0376-7388(00)80317-8

López, O., De La Maza, A., Coderch, L., López-Iglesias, C., Wehrli, E., Parra, J.L., 1998. Direct formation of mixed micelles in the solubilization of phospholipid liposomes by Triton X-100. FEBS Lett. 426, 314-318. https://doi.org/10.1016/S0014-5793(98)003639

Lu, M., Ozcelik, A., Grigsby, C.L., Zhao, Y., Guo, F., Leong, K.W., Huang, T.J., 2016. Microfluidic hydrodynamic focusing for synthesis of nanomaterials. Nano Today 11, 778-792. https://doi.org/10.1016/j.nantod.2016.10.006

Ma, J., Lee, S.M.-Y., Yi, C., Li, C.-W., 2017. Controllable synthesis of functional nanoparticles 660 by microfluidic platforms for biomedical applications - a review. Lab Chip 17, 209-226. https://doi.org/10.1039/C6LC01049K

Maeki, M., Fujishima, Y., Sato, Y., Yasui, T., Kaji, N., Ishida, A., Tani, H., Baba, Y., Harashima, H., Tokeshi, M., 2017. Understanding the formation mechanism of lipid nanoparticles in microfluidic devices with chaotic micromixers. PLoS One 12, 1-16. https://doi.org/10.1371/journal.pone.0187962 
Maeki, M., Kimura, N., Sato, Y., Harashima, H., Tokeshi, M., 2018. Advances in microfluidics for lipid nanoparticles and extracellular vesicles and applications in drug delivery systems. Adv. Drug Deliv. Rev. 128, 84-100. https://doi.org/10.1016/j.addr.2018.03.008

Maeki, M., Saito, T., Sato, Y., Yasui, T., Kaji, N., Ishida, A., Tani, H., Baba, Y., Harashima, H., Tokeshi, M., 2015. A strategy for synthesis of lipid nanoparticles using microfluidic devices with a mixer structure. RSC Adv. 5, 46181-46185. https://doi.org/10.1039/C5RA04690D

Morikawa, Y., Tagami, T., Hoshikawa, A., Ozeki, T., 2018. The Use of an Efficient Microfluidic Mixing System for Generating Stabilized Polymeric Nanoparticles for Controlled Drug Release. Biol. Pharm. Bull. 41, 899-907. https://doi.org/10.1248/bpb.b17-01036

Obeid, M.A., Khadra, I., Mullen, A.B., Tate, R.J., Ferro, V.A., 2017. The effects of hydration media on the characteristics of non-ionic surfactant vesicles (NISV) prepared by microfluidics. Int. J. Pharm. 516, 52-60. https://doi.org/10.1016/j.ijpharm.2016.11.015

680 Patra, M., Salonen, E., Terama, E., Vattulainen, I., Faller, R., Lee, B.W., Holopainen, J., Karttunen, M., 2006. Under the influence of alcohol: The effect of ethanol and methanol on lipid bilayers. Biophys. J. 90, 1121-1135. https://doi.org/10.1529/biophysj.105.062364

Pereira, S., Egbu, R., Jannati, G., Al-Jamal, W.T., 2016. Docetaxel-loaded liposomes: The effect of lipid composition and purification on drug encapsulation and in vitro toxicity. Int. J. Pharm. 514, 150-159. https://doi.org/10.1016/j.ijpharm.2016.06.057

Ran, R., Middelberg, A.P.J., Zhao, C.X., 2016. Microfluidic synthesis of multifunctional liposomes for tumour targeting. Colloids Surfaces B Biointerfaces 148, 402-410. https://doi.org/10.1016/j.colsurfb.2016.09.016 
690 Rothman, K.J., 1990. No adjustments are needed for multiplelrcomparisons. Epidemiology 1, $43-46$.

Rovira-Bru, M., Thompson, D.H., Szleifer, I., 2002. Size and structure of spontaneously forming liposomes in lipid/PEG-lipid mixtures. Biophys. J. 83, 2419-39. https://doi.org/10.1016/S0006-3495(02)75255-7

695 Sabín, J., Prieto, G., Ruso, J.M., Hidalgo-Álvarez, R., Sarmiento, F., 2006. Size and stability of liposomes: A possible role of hydration and osmotic forces. Eur. Phys. J. E 20, 401408. https://doi.org/10.1140/epje/i2006-10029-9

Sackmann, E.K., Fulton, A.L., Beebe, D.J., 2014. The present and future role of microfluidics in biomedical research. Nature 507, 181-189. https://doi.org/10.1038/nature13118

700 Saville, D.J., 1990. Multiple Comparison Procedures: The Practical Solution. Am. Stat. 44, 174. https://doi.org/10.2307/2684163

Simon, S.A., McIntosh, T.J., 1984. Interdigitated hydrocarbon chain packing causes the biphasic transition behavior in lipid/alcohol suspensions. BBA - Biomembr. 773, 169172. https://doi.org/10.1016/0005-2736(84)90562-5

705 Stepto, R.F.T., 2009. Dispersity in polymer science (IUPAC Recommendations 2009). Pure Appl. Chem. 81, 351-353. https://doi.org/10.1351/PAC-REC-08-05-02

Stroock, A.D., 2002. Chaotic Mixer for Microchannels. Science (80-. ). 295, 647-651. https://doi.org/10.1126/science.1066238

Suk, J.S., Hanes, J., Ensign, L.M., Kim, N., Xu, Q., 2015. PEGylation as a strategy for 710 improving nanoparticle-based drug and gene delivery. Adv. Drug Deliv. Rev. 99, 28-51. https://doi.org/10.1016/j.addr.2015.09.012

Suk, J.S., Xu, Q., Kim, N., Hanes, J., Ensign, L.M., 2016. PEGylation as a strategy for 
improving nanoparticle-based drug and gene delivery. Adv. Drug Deliv. Rev. 99, 28-51. https://doi.org/10.1016/j.addr.2015.09.012

715 Takechi-Haraya, Y., Sakai-Kato, K., Abe, Y., Kawanishi, T., Okuda, H., Goda, Y., 2016. Atomic Force Microscopic Analysis of the Effect of Lipid Composition on Liposome Membrane $\quad$ Rigidity. $\quad$ Langmuir $\quad 32, \quad 6074-6082$. https://doi.org/10.1021/acs.langmuir.6b00741

Valencia, P.M., Farokhzad, O.C., Karnik, R., Langer, R., 2012. Microfluidic technologies for accelerating the clinical translation of nanoparticles. Nat. Nanotechnol. 7, 623-629. https://doi.org/10.1038/nnano.2012.168

Vanegas, J.M., Contreras, M.F., Faller, R., Longo, M.L., 2012. Role of unsaturated lipid and ergosterol in ethanol tolerance of model yeast biomembranes. Biophys. J. 102, 507-516. https://doi.org/10.1016/j.bpj.2011.12.038

725 Volpatti, L.R., Yetisen, A.K., 2014. Commercialization of microfluidic devices. Trends Biotechnol. 32, 347-350. https://doi.org/10.1016/j.tibtech.2014.04.010

Whitesides, G.M., 2013. Cool, or simple and cheap? Why not both? Lab Chip 13, 11-13. https://doi.org/10.1039/C2LC90109A

Yanez Arteta, M., Kjellman, T., Bartesaghi, S., Wallin, S., Wu, X., Kvist, A.J., Dabkowska, A., Székely, N., Radulescu, A., Bergenholtz, J., Lindfors, L., 2018. Successful reprogramming of cellular protein production through mRNA delivered by functionalized lipid nanoparticles. Proc. Natl. Acad. Sci. 115, E3351-E3360. https://doi.org/10.1073/pnas.1720542115

Yu, B., Lee, R.J., Lee, L.J., 2009. Microfluidic Methods for Production of Liposomes. Methods Enzymol. 465, 129-141. https://doi.org/10.1016/S0076-6879(09)65007-2 
Zetterberg, M.M., Ahlgren, S., Agmo Hernández, V., Parveen, N., Edwards, K., 2016. Optimization of lipodisk properties by modification of the extent and density of the PEG corona. J. Colloid Interface Sci. 484, 86-96. https://doi.org/10.1016/j.jcis.2016.08.067

Zheng, M., Fyles, T.M., 2018. Properties of Liposomes Containing Natural and Synthetic Lipids Formed by Microfluidic Mixing. Eur. J. Lipid Sci. Technol. 120, 1-8. https://doi.org/10.1002/ejlt.201700347

Zhigaltsev, I. V., Belliveau, N., Hafez, I., Leung, A.K.K., Huft, J., Hansen, C., Cullis, P.R., 2012. Bottom-up design and synthesis of limit size lipid nanoparticle systems with aqueous and triglyceride cores using millisecond microfluidic mixing. Langmuir 28, 3633-3640. https://doi.org/10.1021/la204833h

Zhigaltsev, I. V., Tam, Y.K., Leung, A.K.K., Cullis, P.R., 2015. Production of limit size nanoliposomal systems with potential utility as ultra-small drug delivery agents. J. Liposome Res. 00, 1-7. https://doi.org/10.3109/08982104.2015.1025411

Zizzari, A., Bianco, M., Carbone, L., Perrone, E., Amato, F., Maruccio, G., Rendina, F., Arima, V., 2017. Continuous-flow production of injectable liposomes via a microfluidic approach. Materials (Basel). 10, 1-13. https://doi.org/10.3390/ma10121411

Zook, J.M., Vreeland, W.N., 2010. Effects of temperature, acyl chain length, and flow-rate ratio on liposome formation and size in a microfluidic hydrodynamic focusing device. Soft Matter 6, 1352. https://doi.org/10.1039/b923299k 


\section{Tables}

Table 1. Lipid composition of formulations prepared in this study.

\begin{tabular}{lll}
\hline Formulation & Lipid Composition & Molar Ratio \\
\hline DOPC5 & DOPC/Chol/DSPE-PEG 2000 & $95 / 50 / 5$ \\
DPPC5 & DPPC/Chol/DSPE-PEG 2000 & $95 / 50 / 5$ \\
DSPC5 & DSPC/Chol/DSPE-PEG 2000 & $95 / 50 / 5$ \\
DSPC4 & DSPC/Chol/DSPE-PEG 2000 & $96 / 50 / 4$ \\
DSPC2.5 & DSPC/Chol/DSPE-PEG 2000 & $97.5 / 50 / 2.5$ \\
DSPC0 & DSPC/Chol & $100 / 50$
\end{tabular}


760 Table 2. Optimised flow conditions, corresponding hydrodynamic diameter and dispersity of sterically stabilised liposomal formulations for stability study (with initial lipid concentration of $10 \mathrm{mM}$ ) prepared in different aqueous media (NS, PBS (pH 7.4), HBS (pH 7.4), and $\left(\mathrm{NH}_{4}\right)_{2} \mathrm{SO}_{4}(\mathrm{pH}$ 5.4)) by the SHM device. Data represents the mean \pm SD of at least three independent experiments.

\begin{tabular}{ccccccc}
\hline \multirow{2}{*}{ Formulation } & \multicolumn{2}{c}{$\begin{array}{c}\text { Optimised flow } \\
\text { conditions }\end{array}$} & \multicolumn{4}{c}{ Hydrodynamic diameter (nm) } \\
& TFR (mL/min) & FRR & NS & PBS & HBS & $\left(\mathrm{NH}_{4}\right)_{2} \mathrm{SO}_{4}$ \\
\hline \multirow{2}{*}{ DOPC5 } & 2 & 1 & $101.5 \pm 5.1$ & $116.8 \pm 7.9$ & $100.91 \pm 8.4$ & $120.7 \pm 8.6$ \\
& & & $0.10 \pm 0.01$ & $0.10 \pm 0.02$ & $0.09 \pm 0.01$ & $0.10 \pm 0.03$ \\
DSPC2.5 & 2 & 3 & $122.0 \pm 8.8$ & $111.4 \pm 5.8$ & $116.1 \pm 3.5$ & $136.6 \pm 5.3$ \\
& & & $0.06 \pm 0.01$ & $0.09 \pm 0.02$ & $0.09 \pm 0.01$ & $0.11 \pm 0.01$ \\
DSPC5 & 2 & 3 & $100.0 \pm 8.6$ & $85.81 \pm 9.3$ & $110.4 \pm 6.7$ & $106.2 \pm 4.30$ \\
& & & $0.26 \pm 0.02$ & $0.25 \pm 0.08$ & $0.29 \pm 0.04$ & $0.23 \pm 0.05$ \\
DPPC5 & 2 & 3 & $107.4 \pm 3.8$ & $92.45 \pm 7.8$ & $111.7 \pm 4.3$ & $118.1 \pm 12.3$ \\
& \multirow{2}{*}{2} & 3 & $0.10 \pm 0.02$ & $0.11 \pm 0.02$ & $0.09 \pm 0.01$ & $0.09 \pm 0.01$ \\
\hline
\end{tabular}


Table 3. Modified initial lipid concentration, optimised flow conditions, corresponding hydrodynamic diameter, dispersity, $\zeta$-potential, and DOX encapsulation efficiency (EE) of DOPC-, DSPC-, DPPCbased liposomal formulations prepared in (NH4)2SO4 (pH 5.4) buffer and remotely-loaded with DOX.

\begin{tabular}{|c|c|c|c|c|c|c|c|}
\hline $\begin{array}{l}\text { Formulati } \\
\text { on }\end{array}$ & $\begin{array}{l}\text { Initial lipid } \\
\text { concentration }\end{array}$ & $\begin{array}{l}\text { Optimi } \\
\text { cond } \\
\text { TFR } \\
(\mathrm{mL} / \mathrm{m} \\
\text { in })\end{array}$ & $\begin{array}{l}\text { ed flow } \\
\text { tions } \\
\text { FRR }\end{array}$ & $\begin{array}{c}\text { Z- } \\
\text { Average } \\
(\mathbf{n m})\end{array}$ & Dispersity & $\begin{array}{c}\zeta- \\
\text { potential } \\
(\mathbf{m V})\end{array}$ & $\% \mathbf{E E}$ \\
\hline DOPC5 & $15 \mathrm{mM}$ & 2 & 1 & $\begin{array}{l}125.1 \pm \\
10.8\end{array}$ & $0.12 \pm 0.03$ & $\begin{array}{l}-12.3 \pm \\
2.5\end{array}$ & $\begin{array}{l}88.2 \pm \\
3.2\end{array}$ \\
\hline DSPC2.5 & $30 \mathrm{mM}$ & 2 & 3 & $\begin{array}{l}131.5 \pm \\
9.6\end{array}$ & $0.11 \pm 0.03$ & $-8.7 \pm 1.4$ & $\begin{array}{l}84.9 \pm \\
4.5\end{array}$ \\
\hline DSPC5 & $30 \mathrm{mM}$ & 2 & 3 & $\begin{array}{l}115.4 \pm \\
0.6\end{array}$ & $0.20 \pm 0.01$ & $\begin{array}{l}-11.8 \pm \\
0.6\end{array}$ & $\begin{array}{l}88.4 \pm \\
2.5\end{array}$ \\
\hline DPPC5 & $30 \mathrm{mM}$ & 2 & 3 & $\begin{array}{l}134.0 \pm \\
4.9\end{array}$ & $0.10 \pm 0.04$ & $\begin{array}{l}-10.3 \pm \\
1.6\end{array}$ & $\begin{array}{l}82.0 \pm \\
5.4\end{array}$ \\
\hline
\end{tabular}




\section{Figure and scheme captions}

Scheme 1. Schematic of the setup for microfluidic preparation of liposomes using a staggered herringbone micromixer (SHM).

Figure 1. The effect of the aqueous media on DOPC5 size and dispersity. Z-Average diameter and dispersity of $10 \mathrm{mM}$ of DOPC5 prepared in DW, NS and PBS, with TFR of 1 and $2 \mathrm{~mL} / \mathrm{min}$, and FRR of 1. Solid bars and open circles $(O)$ indicate the liposomes Z-Average diameter and dispersity, respectively. The data represents the mean \pm SD of at least three independent experiments. All differences between means, with $\mathrm{p}<0.05$ are indicated (LSD test), in comparison to DW of the same TFR. **, $\mathrm{p}<0.01$ comparing Z-Average diameter. \#, $\mathrm{p}<0.05$;

$780 \quad \#, \mathrm{p}<0.01 ; \# \#, \mathrm{p}<0.001$ comparing dispersity.

Figure 2. The effect of TFR and FRR on DOPC5 size and dispersity. Z-Average diameter and dispersity of $10 \mathrm{mM}$ of DOPC5 prepared in (a) NS and (b) PBS, with TFR of 1 and $2 \mathrm{~mL} / \mathrm{min}$ and FRR of 1, 2, 3 and 5. Solid bars and open circles $(\bigcirc)$ indicate the liposomes Z-Average diameter and dispersity, respectively. The data represents the mean \pm SD of at least three independent experiments. All differences between means, with $\mathrm{p}<0.05$ are indicated (t-test), comparing TFR with the same FRR. *, p < 0.05; **, p < $0.01 ; * * *, p<0.001$ comparing ZAverage diameter.

Figure 3. The effect of initial lipid concentration on DOPC5 size and dispersity. Z-Average diameter and dispersity of 5,10,15 mM of DOPC5 prepared in NS and PBS, with TFR of 2 $\mathrm{mL} / \mathrm{min}$ and FRR of 1 . Solid bars and open circles $(\mathrm{O})$ indicate the liposomes Z-Average diameter and dispersity, respectively. The data represents the mean \pm SD of at least three independent experiments. All differences between means with $\mathrm{p}<0.05$ are indicated (LSD test), in comparison with $10 \mathrm{mM} . \#, \mathrm{p}<0.05$; \#\#, $\mathrm{p}<0.01$ comparing dispersity. 
Figure 4. The effect of FRR on DSPC5 size and dispersity. Z-Average diameter and dispersity of $10 \mathrm{mM}$ of DSPC5 prepared in NS or PBS, with TFR of $2 \mathrm{~mL} / \mathrm{min}$ and FRR of 2, 2.5, 3 and 4. Solid bars and open circles $(\bigcirc)$ indicate the liposomes Z-Average diameter and dispersity, respectively. The data represents the mean \pm SD of at least three independent experiments.

Figure 5. The effect of DSPE-PEG 2000 content on DSPC/Chol/DSPE-PEG $2000(100-\mathrm{x} / 50 / \mathrm{x}, \mathrm{x}$ $=0-5)$ liposomes size and dispersity. Z-Average diameter and dispersity of $10 \mathrm{mM}$ DSPC/Chol/DSPE-PEG $2000(100-\mathrm{x} / 50 / \mathrm{x}, \mathrm{x}=0-5)$ prepared in NS and PBS, with TFR of 2 $\mathrm{mL} / \mathrm{min}$ and FRR of 3. Solid bars and open circles $(\mathrm{O})$ indicate the liposomes Z-Average diameter and dispersity, respectively. The data represents the mean $\pm \mathrm{SD}$ of at least three independent experiments. All differences between means, in comparison to 5 mol\% DSPE$\mathrm{PEG}_{2000}$ of the same aqueous medium, with $\mathrm{p}<0.05$ are indicated (LSD test). *, $\mathrm{p}<0.05 ; * *$, $805 \mathrm{p}<0.01$ comparing Z-Average diameter. \#, $\mathrm{p}<0.05$; \#\#, $\mathrm{p}<0.01$; \#\#, $\mathrm{p}<0.001$ comparing dispersity.

Figure 6. The effect of phospholipid rigidity on liposomes size and dispersity. Z-Average diameter and dispersity of $10 \mathrm{mM}$ DOPC5, DPPC5 and DSPC5 prepared in NS and PBS, with TFR of $2 \mathrm{~mL} / \mathrm{min}$ and FRR of 3. Solid bars and open circles $(O)$ indicate the liposomes Z-

810 Average diameter and dispersity, respectively. The data represents the mean \pm SD of at least three independent experiments. All differences between means, in comparison DSPC5 of the same aqueous medium, with $\mathrm{p}<0.05$ are indicated (LSD test). ${ }^{*}, \mathrm{p}<0.05 ; * *, \mathrm{p}<0.01$ comparing Z-Average diameter. \#, $\mathrm{p}<0.05$; \#\#, $\mathrm{p}<0.01$; \#\#, $\mathrm{p}<0.001$ comparing dispersity.

Figure 7. Structural elucidation of microfluidics prepared liposomes. Transmission electron 815 microscopy (TEM) images of (a) DOPC5, (b) DSPC2.5, and (c) DPPC5 prepared with their 
respective optimised flow conditions, as described in Table 2, in NS. Liposomes prepared have vesicular structures with diameter of around $100 \mathrm{~nm}$. Scale bar represents (a) $200 \mathrm{~nm}$, and (b, c) $100 \mathrm{~nm}$.

Scheme 2. Flowchart for designing and optimisation a liposomal formulation using SHM. The 820 flowchart is arranged, from top to bottom, based on the influence of each parameter on the property of the liposomal formulation. The qualitative effect of increasing respective parameter on the size and dispersity of liposomes prepared by microfluidics is indicated. 